\title{
Nonlinear self-phase matching of optical second harmonic generation in lithium niobate
}

\author{
Sergei Orlov and Amnon Yariv \\ California Institute of Technology, 128-95, Pasadena, California 91125 \\ Mordechai Segev \\ Department of Electrical Engineering, Princeton University, Princeton, New Jersey 08544
}

(Received 8 November 1995; accepted for publication 22 January 1996)

\begin{abstract}
We show that the nonlinear index perturbation due to light-induced photovoltaic space-charge field in $\mathrm{LiNbO}_{3}$ can give rise to self-phase matching of second harmonic generation. Increase of the conversion efficiency is accompanied by formation of stationary and nonstationary patterns in the spatial structure of the generated second harmonic. The space-charge field can be induced either by the initially non-phase-matched second harmonic or by an external seed. (C) 1996 American Institute of Physics. [S0003-6951(96)04412-9]
\end{abstract}

Different techniques for phase matching the nonlinear interactions of optical beams have been developed including temperature and angular tuning for birefringent phase matching and quasiphase matching ${ }^{1}$ using an alternating sign optical nonlinearity.

Photorefraction [optical damage (Refs. 2 and 3) in $\left.\mathrm{LiNbO}_{3}\right]$ is, in general, a negative feature for optical second harmonic generation (SHG) applications, since it locally modifies the birefringence of the crystal, thus, changing the phase-matching conditions for SHG dramatically. We demonstrate that the large nonlinear index perturbation due to photovoltaic effect can promote a new effect, namely, the self-phase matching of SHG (initially nonphase matched) which manifests itself in a large increase in conversion efficiency and pattern formation in the structure of the generated SH beam. ${ }^{4}$

As an optical source, we employ a $Q$-switched (repetition rate $1 \mathrm{kHz})$ mode-locked $(50 \mathrm{MHz}) \mathrm{Nd}$ :YAG laser $(\lambda$ $=1.064 \mu \mathrm{m})$, emitting $8-10$ mode-locked 100 ps pulses within the $Q$-switched envelope. We focus (Fig. 1, inset) the ordinarily polarized IR radiation with a $50 \mathrm{~mm}$ focal length lens on a lightly $\mathrm{Fe}$-doped $2 \mathrm{~mm}$ thick $\mathrm{LiNbO}_{3}$ crystal, the $c$ axis of which is in the plane of incidence. The focal spot size of the infrared beam is $\sim 70 \mu \mathrm{m}$. The extraordinarily polarized second harmonic light $(\lambda=0.532 \mu \mathrm{m})$ emerging from the far side of the crystal is captured by a charge coupled device (CCD) camera and its total $\mathrm{cw}$ power is measured with a photodetector.

We observe that for relatively small angles of incidence (between $0^{\circ}$ and approximately $20^{\circ}$ ) of the fundamental light the second harmonic signal increases with time (Fig. 1) by a factor $\sim 100$ from a few $\mu \mathrm{W}$ to several $\mathrm{mW}$ average power (up to $0.1 \%$ in conversion efficiency). The large enhancement in the $\mathrm{SH}$ power is accompanied by a pattern formation in the spatial structure of the SH beam (Fig. 2). The SH beam first becomes elongated along the direction of the $c$ axis of the crystal, has two distinct side lobes in the intermediate stage [Fig. 2(b)], and eventually settles into the pattern of Fig. 2(c). At the same time, the ordinarily polarized infrared beam does not experience substantial phase distortion, as seen from its far-field pattern, which remains roughly a Gaussian. The enhancement in the SHG can also be induced by the green $(\lambda=0.532 \mu \mathrm{m})$ seeding beam alone. If a spot previously exposed to the externally injected seeding $\mathrm{SH}$ light (focused with the same lens) is probed by the infrared light the initially $(t=0)$ generated SH signal is significantly (50-100 times) higher and the time for the SH signal to reach the final steady-state intensity is shorter than in a "fresh" spot. This is in contrast to the self-induced SHG in glass fibers ${ }^{5}$ where the simultaneous presence of the fundamental and SH (either self-induced or injected) light is required by the nature of the $\chi^{(3)}$ coherent photovoltaic effect. ${ }^{6}$ The photoinduced index changes persist for prolonged periods of time when the crystal is kept in the dark, but can be completely erased optically by homogeneous prolonged illumination with visible or UV light. This, along with the anisotropy of the SH far-field pattern (Fig. 2) indicate that the effect is not of thermal origin (since thermally induced refractive index changes are isotropic, as emphasized in Ref. 7). Furthermore, the index mismatch for SHG at $1.064 \mu \mathrm{m}$ increases with temperature in congruent $\mathrm{LiNbO}_{3}$, thus, temperature increase cannot phase match or enhance the SHG process.

We conclude that the origin of the self-phase-matching effect is the photovoltaic field induced by the second harmonic light (initially nonphase matched) which via conventional electrooptic effect locally changes the birefringence of

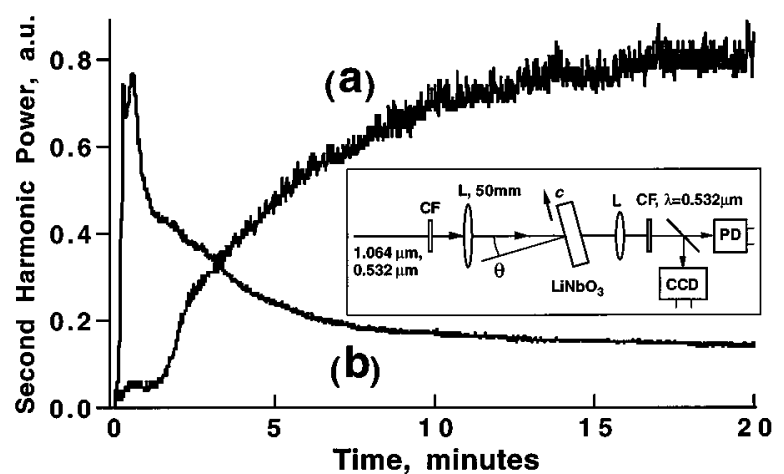

FIG. 1. Growth of $\mathrm{SH}$ with time (a) for $16^{\circ}$ angle of incidence, (b) for normal incidence. Optical power at $1.064 \mu \mathrm{m}$ is $1.5 \mathrm{~W}$. Inset: experimental setup. $\mathrm{L}$ is a lens, PD is a photodetector, CF's are appropriate color filters. 

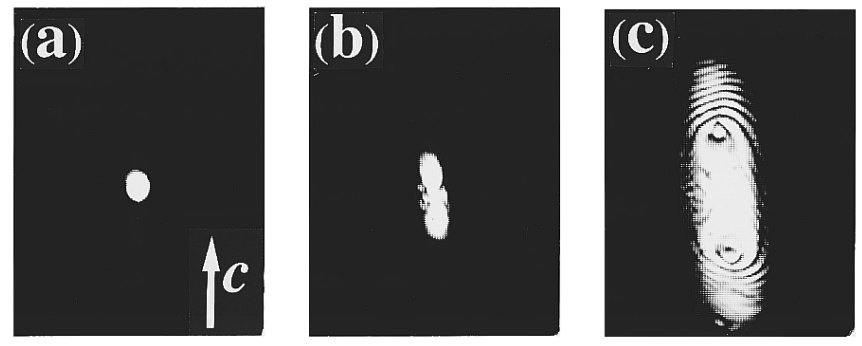

FIG. 2. Far field of the generated $\mathrm{SH}$ beam in a $2 \mathrm{~mm}$ crystal (a) at the beginning of exposure, (b) intermediate stage, (c) at saturation (total far-field angle of the beam in the $c$-axis plane is $\sim 25^{\circ}$ ).

the crystal to provide (or improve significantly) the index matching, resulting in the increase of the generated $\mathrm{SH}$ power. Fe-doped $\mathrm{LiNbO}_{3}$ has very low photosensitivity in the near IR but exhibits strong photovoltaic response in the visible range (for $\lambda \leqslant 0.5 \mu \mathrm{m}$ ). The maximal optically induced perturbation (negative by sign) in the extraordinary index of refraction is typically $\sim 0.001$ and is larger the higher the light intensity, ${ }^{8}$ which is consistent with the mismatch value ${ }^{9} \Delta n=n_{e}^{2 \omega}-n_{0}^{\omega} \cong 0.002$ for SHG at $\lambda=1.064$ $\mu \mathrm{m}$. The far-field pattern [Figs. 3(b) and 3(c)] is caused by the spatial self-phase modulation (self-defocusing) owing to the nonlinear index change induced due to the photovoltaic field by the nonuniform spatial profile of the SH beam. The pattern differs from the conventional picture of conical rings [typical for spatial self-phase modulation of isotropic origin (Ref. 7)] because of the anisotropy of the index perturbation arising due to photovoltaic effect. Spatial profile of $\Delta n_{e}$ (Fig. 3 , left) generated by a Gaussian beam is obtained by solving numerically nonlinear Kukhtarev's equations (i.e., rate and continuity equation including photovoltaic current, and Gauss law) in two transverse dimensions. ${ }^{10}$ The $c$-axis projection of the space-charge field is maximal and has nearly flat profile in the center of the beam, has two side lobes on its margins, and falls off to zero value where the light intensity is less than the effective dark irradiance $I(r) \ll I_{d} .{ }^{11}$ At the same time, the gradients of $\Delta n_{e}$ are the largest along the direction of the $c$ axis of the crystal. This results in preferential scattering of the SH beam in the $c$-axis plane. Figure 3,

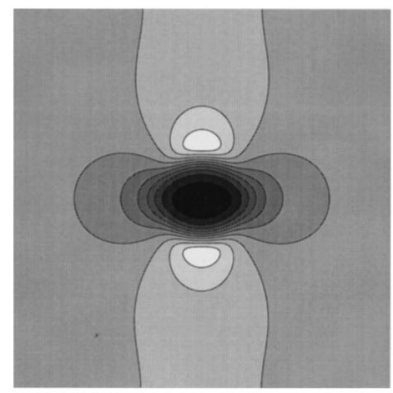

(a)

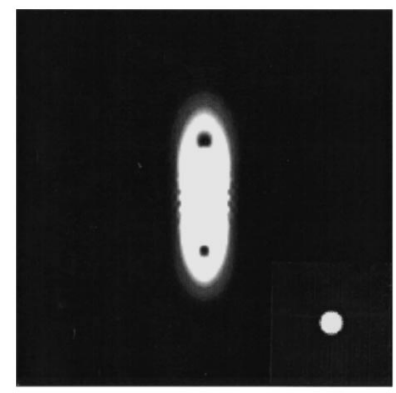

(b)
FIG. 3. Left, extraordinary index perturbation $\Delta n_{e}$ induced by a Gaussian beam (dark regions correspond to negative index change). Beam diameter $d$ is $1 / 6$ of the size of computation region, $I_{\text {dark }}=0.05 I_{00}$, characteristic diffusion field $k_{b}$ T/ed $=0.01 E_{p . v \text {. }}$, trap saturation is neglected (Ref. 10). Right, corresponding far-field pattern of the same Gaussian for weak index perturbation $\left|\Delta n_{e}(\max )\right| L / \lambda \approx 3$. Diffraction within the crystal is neglected. Note a slight asymmetry in $+/-c$ direction due to diffusion field. $c$-axis direction is vertical. Inset: far field of unperturbed beam (same scale).

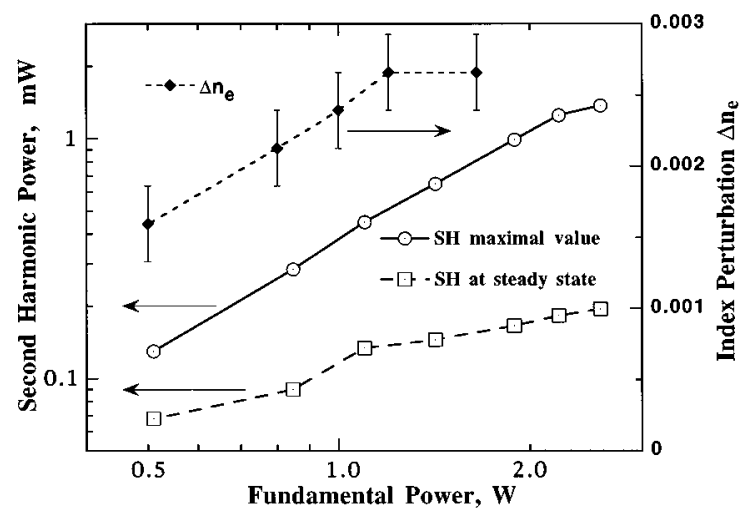

FIG. 4. Second harmonic power at steady state (after $2000 \mathrm{~s}$ of exposure) and at maximum, and the magnitude of the extraordinary index change $\Delta n_{e}$ vs the incident power of the fundamental beam (normal incidence, $\left.\theta=0^{\circ}\right)$.

right shows that even in the case of a round Gaussian input beam its far field is strongly and asymmetrically distorted along the $c$-axis direction.

The magnitude of extraordinary index change is estimated using an approximate formula ${ }^{7} \Delta n_{e} L \approx N \lambda$, where $N$ is the number of the orders of diffraction (interference fringes), observed in the far field, and $L$ is the length of the crystal (Fig. 4). From Fig. 2(c) we obtain $N \approx 9$, therefore, $\Delta n_{e} \approx-2.6 \times 10^{-3}$. The perturbation in the ordinary index for the fundamental light is much smaller since $r_{13}$ (at 1.064 $\mu \mathrm{m}) / r_{33}($ at $0.532 \mu \mathrm{m}) \approx 0.2$ in $\mathrm{LiNbO}_{3}$. This explains the absence of patterns in the far field of the infrared beam. The $\Delta n_{e}$ changes with input power (Fig. 4) indicating that in the range of SH intensities achieved in the experiments, the photovoltaic field is essentially intensity dependent. ${ }^{8}$ Therefore, the extraordinary index change and, thus, the phase matching conditions are different for different input powers. This explains the deviation of the $\mathrm{SH}$ power in the steady state from the expected square law $P_{2 \omega} \propto P_{\omega}^{2}$. In general, the temporal evolution of the SH signal is not a simple exponent [see Fig. 1(b)], however, the characteristic response time $\tau$ of the buildup process, defined as the time required for the SH signal to reach a half of its maximum value, scales with input power as $\tau \propto P_{\omega}^{-2.4 \pm 0.2}$, when other parameters are kept the same.

For a particular initial index mismatch the generated photovoltaic fields may be larger than required to achieve the ideal phase matching, i.e., in some cases the light-induced index change over compensates. This results in the decrease of the SH power after its initial rapid buildup [Fig. 1(b)]. The SH signal does not return to a near zero value due to the strong defocusing channel induced in the medium. Part of the nonideally phase-matched second harmonic leaves the interaction region before being converted back to the fundamental beam. This is somewhat analogous to the Cherenkovtype SHG in waveguides. ${ }^{12}$ The SHG process can be optimized by adjusting the initial $(t=0)$ index mismatch of the interacting waves at any particular input power level. Figure 5 shows such angular tuning curve for SHG. The optimal angle of incidence (14 $4^{\circ}$ in the conditions of Fig. 5) may differ for other Fe-doping concentrations, temperatures, and may also depend on input intensity. Using the Sellmeier 




FIG. 5. Second harmonic power at steady state (after $20 \mathrm{~min}$ ) (circles, solid line) and the buildup time $\tau$ (squares, dashed line) vs incidence angle (in air) of the fundamental beam (optical power $1.5 \mathrm{~W}$, lower scale) and the initial $\Delta n=n_{e}^{2 \omega}-n_{0}^{\omega}$ calculated using Sellmeier equation (upper scale). The $\mathrm{SH}$ power obtained with a critically phase-matched $\mathrm{Mg}$-doped $\mathrm{LiNbO}_{3}$ crystal is $4.2 \mathrm{~mW}$ in the same experimental arrangements.

equation ${ }^{9}$ for undoped congruent $\mathrm{LiNbO}_{3}$ we evaluate the maximal $\Delta n=n_{e}^{2 \omega}-n_{0}^{\omega}$ which can be compensated to be $\Delta n$ $\approx 0.003$. This corresponds to the optically induced electric space-charge field of order of $\sim 250 \mathrm{kV} / \mathrm{cm}$.

The characteristic response time $\tau$ of the phase-matching process depends significantly on the starting index mismatch, which is controlled by changing the incidence angle (Fig. 5). The response time has a maximum in the vicinity of the optimal angle and decreases otherwise, rather than is a monotonically increasing function of the starting mismatch $n_{e}^{2 \omega}(t=0)-n_{0}^{\omega}$. This behavior is a characteristic of "critical slowing down" phenomena relevant to other self-organizing systems which exhibit pattern formation. ${ }^{13}$ The self-phasematched SHG may also be viewed as a self-organizing process with the order parameter being the difference between the induced index perturbation and the one required to achieve the ideally phase matched SHG.

The self-phase-matching effect was found in a large variety of oxidized Fe-doped $\mathrm{LiNbO}_{3}$ samples. The temporal evolution of the spatial structure of the emerging second harmonic in a $5 \mathrm{~mm}$ long Fe-doped $(0.05 \mathrm{wt} \%)$ crystal is shown in Fig. 6. Similarly to Fig. 2, the initially weak Gaussian SH beam first "emits" two side lobes, which split further [Fig. 6(a)]. After the power of the second harmonic builds up the pattern with multiple orders of diffraction [Fig. 6(b)] establishes itself. The further evolution is different from what was observed previously in a short plate sample. The pattern continues to evolve in time into a picture of random moving speckles [Fig. 6(c)], where no large scale structures can be resolved. The latter is somewhat similar to the conventional
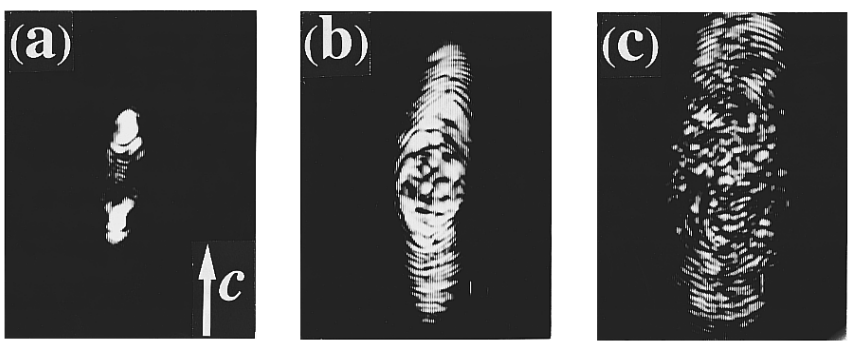

FIG. 6. Far field of the generated $\mathrm{SH}$ beam in a $5 \mathrm{~mm}$ long crystal (a) intermediate stage ( $t=20 \mathrm{~s}$ after the beginning of exposure), (b) pattern with multiple orders of diffraction $(t=100 \mathrm{~s})(\mathrm{c})$ after the speckled structure has developed ( $t=400 \mathrm{~s}$; total far-field angle of the beam in the $c$-axis plane is $\sim 40^{\circ}$ ).

picture of optical damage in $\mathrm{LiNbO}_{3},{ }^{2,3}$ where, unlike the SHG case, the whole issue of phase matching is irrelevant. It is yet to be determined whether the observed nonstationary state corresponds to the transition of the system to the optical chaos, or is caused by local changes in the refractive index due to material effects (e.g., due to thermooptic effect).

In conclusion, we have demonstrated self-phase matching of optical SHG due to nonlinear index perturbation. In the presence of strong photovoltaic effect in $\mathrm{LiNbO}_{3}$ the SHG nonlinear interaction manifests itself in two characteristic and very apparent manners: a large change in the conversion efficiency and self-induced transversal beam structure (stationary or nonstationary) of the generated second harmonic.

This work is supported by the Advanced Research Project Agency, the Army Research Office, and the National Institute of Standards and Technology.

${ }^{1}$ J. A. Armstrong, N. Bloembergen, J. Ducuing, and P. S. Pershan, Phys. Rev. 127, 1918 (1962); M. M. Fejer, G. A. Magel, D. H. Jundt, and R. L. Byer, IEEE J. Quantum Electron. QE-28, 2631 (1992)

${ }^{2}$ F. S. Chen, J. Appl. Phys. 40, 3389 (1969).

${ }^{3}$ A. Ashkin, G. D. Boyd, J. M. Dziedzic, R. G. Smith, A. A. Ballman, J. J. Levinstein, and K. Nassau, Appl. Phys. Lett. 9, 72 (1966).

${ }^{4}$ S. Orlov, A. Yariv, and M. Segev, in Conference on Lasers and ElectroOptics, Vol. 15 of 1995 OSA Technical Digest Series (Optical Society of America, Washington, D.C., 1995), CWK2, p. 243.

${ }^{5}$ U. Österberg and W. Margulis, Opt. Lett. 11, 516 (1986).

${ }^{6}$ E. M. Dianov, P. G. Kazansky, and D. Yu. Stepanov, Sov. J. Quantum Electron. 19, 575 (1989).

${ }^{7}$ S. D. Durbin, S. M. Arakelian, and Y. R. Shen, Opt. Lett. 9, 411 (1981); M. Horowitz, R. Daisy, O. Werner, and B. Fischer, ibid. 17, 475 (1992).

${ }^{8}$ I. F. Kanaev, V. K. Malinovsky, and A. M. Pugachev, Ferroelectrics 75, 209 (1987); F. Jermann and J. Otten, J. Opt. Soc. Am. B 10, 2085 (1993).

${ }^{9}$ D. S. Smith, H. D. Riccius, and R. P. Edmin, Opt. Commun. 17, 332 (1976).

${ }^{10}$ A. A. Zozulya and D. Z. Anderson, Phys. Rev. A 51, 1520 (1995).

${ }^{11}$ G. C. Valley, M. Segev, B. Crosignani, A. Yariv, M. M. Fejer, and M. C. Bashaw, Phys. Rev. A 50, R4457 (1994).

${ }^{12}$ P. K. Tien, R. Ulrich, and R. Martin, Appl. Phys. Lett. 17, 447 (1970).

${ }^{13}$ M. C. Cross and P. C. Hohenberg, Rev. Mod. Phys. 65, 851 (1993). 